\title{
An explainable supervised machine learning predictor of acute kidney injury after adult deceased donor liver transplantation
}

Yihan Zhang ( $\sim$ zhyihan@mail3.sysu.edu.cn)

The Third Affiliated Hospital of Sun Yat-Sen University https://orcid.org/0000-0002-3546-9752

Dong Yang

Guangzhou AID cloud technology co., LTD

\section{Zifeng Liu}

The Third Affiliated Hospital of Sun Yat-sen University

\section{Chaojin Chen}

The Third Affiliated Hospital of Sun Yat-sen University

\section{Mian Ge}

The Third Affiliated Hospital of Sun Yat-sen University

\section{Xiang Li}

The Third Affiliated Hospital of Sun Yat-sen University

\section{Tongsen Luo}

The Third Affiliated Hospital of Sun Yat-sen University

\section{Zhengdong Wu}

Guangzhou AID cloud technology co. LTD

\section{Chenguang Shi}

Guangzhou AID cloud technology co., LTD

\section{Bohan Wang}

Guangzhou AID cloud technology co., LTD

\section{Xiaoshuai Huang}

Guangzhou AID cloud technology co., LTD

\section{Xiaodong Zhang}

The Third Affiliated Hospital of Sun Yat-sen University

\section{Shaoli Zhou}

The Third Affiliated Hospital of Sun Yat-sen University

\section{Ziqing Hei}

The Third Affiliated Hospital of Sun Yat-sen University 
Keywords: kidney dysfunction, liver transplant, SHarpley Additive exPlaination methods, SHAP value, gradient boosting machine, perioperative medicine, big data, artificial intelligence, prognostic predictor, clinical assisting tool

Posted Date: April 30th, 2021

DOI: https://doi.org/10.21203/rs.3.rs-442049/v1

License: (c) (1) This work is licensed under a Creative Commons Attribution 4.0 International License. Read Full License

Version of Record: A version of this preprint was published at Journal of Translational Medicine on July 28th, 2021. See the published version at https://doi.org/10.1186/s12967-021-02990-4. 


\section{Abstract}

Background: Early prediction of acute kidney injury (AKI) after liver transplantation (LT) facilitates timely recognition and intervention. We aimed to build a risk predictor of post-LT AKI via supervised machine learning and visualize the mechanism driving within to assist clinical decision-making.

Methods: Data of 894 cases that underwent liver transplantation from January 2015 to September 2019 were collected, covering demographics, donor characteristics, etiology, peri-operative laboratory results, co-morbidities and medications. The primary outcome was new-onset AKI after LT according to Kidney Disease Improving Global Outcomes guidelines. Predicting performance of five classifiers including logistic regression, support vector machine, random forest, gradient boosting machine (GBM) and adaptive boosting were respectively evaluated by the area under the receiver-operating characteristic curve (AUC), accuracy, F1-score, sensitivity and specificity. SHapley Additive exPlanations (SHAP) method was applied to evaluate feature importance and explain the predictions made by $\mathrm{ML}$ algorithms.

Results: 430 AKI cases (55.1\%) were diagnosed out of 780 included cases. The GBM model achieved the highest AUC $(0.76, \mathrm{Cl} 0.70$ to 0.82$)$, F1-score $(0.73, \mathrm{Cl} 0.66$ to 0.79$)$ and sensitivity $(0.74, \mathrm{Cl} 0.66$ to 0.8$)$. High preoperative indirect bilirubin, low intraoperative urine output, long anesthesia time, low preoperative platelets, and graft steatosis graded NASH CRN 1 and above were revealed by SHAP method the top 5 important variables contributing to the diagnosis of post-LT AKI made by GBM model.

Conclusions: Our GBM-based predictor of post-LT AKI provides a highly interoperable tool across institutions to assist decision-making after LT.

\section{Introduction}

Acute kidney injury (AKI) after liver transplantation (LT) holds unique etiology and risk factors compared to AKI in other clinical settings. The estimated incidence of AKI following LT varies from $17 \%$ to $95 \%(1$, 2), with an average around $40.7 \%$ (3) [1, 2]. AKI after LT is associated with increased post-operative mortality, potential progression to chronic kidney disease (CKD), longer length of stay and increased medical expenditure(1).Graft characteristics, intraoperative hemodynamic instability and post-operative exposure to nephrotoxic immunosuppression have been considered to be associated with AKI after LT(46). Early interventions like perioperative continuous renal replacement therapy (CRRT)and restraint on nephrotoxic medications shall be considered in patients with AKI, but the timing of such decisions depends largely on personal experience and a reliable predicting model can greatly facilitate these decisions(7).

Machine learning (ML) algorithms have demonstrated satisfactory performance in building robust predictive models of inpatient AKI(8). However, many of these studies fed relatively abundant features to $\mathrm{ML}$ algorithms without dimensionality reduction (9). Highly correlated features without regularization are of limited utility in enhancing the predictive power of the model(10). Moreover, high dimensional features are susceptible to missing data once being externally validated across institutions, hindering clinical 
application of these models. With current surge of these ML-derived clinical assisting tool $(11,12)$, criteria for evaluation and regulation of such predictive algorithms have been advocated, which include setting meaningful endpoints and appropriate benchmarks, and ensuring generalizability among institutions(13).

Besides these criteria, relational validity of ML-derived predictive models, that is, the extent to which physicians can interpret them, has been emphasized lately, since a sound statistical validity does not necessarily guarantee the usability of these models(14). The "black magic" of ML remains to be debated for the difficulty to understand the mechanisms driving within(15). SHapley Additive exPlanations (SHAP) method developed by Lundberg(16)is a Game Theory-based method, within which the individual features act as players in a prediction task and the Shapley value helps to fairly distribute the prediction performance among the features(17). This method enables black-box ML algorithms to be explained on individual level. In this study we aimed to select a ML classifier that outperform statistically in predicting post-LT AKI and further visualize the decision made $M L$ algorithms to clinicians to assist their decisions. Meanwhile we also validated an AKI prediction score developed by Kalisvaart et al.(5) with our data set and compared the performance of our ML model to this score.

\section{Experimental Procedures}

Source of Data and Participants

This was a retrospective, single center research conducted in The Third Affiliated Hospital of Sun Yat-sen University-Lingnan Hospital. This study was approved by the Ethnic Committee of the Third Affiliated Hospital of Sun Yat-sen University (NO. [2019]02-609-01), with waiver of informed consent.

Medical data collected by natural language process module from EMRs included demographic data, daily documentation, laboratory and imaging results, anesthesia records, medications, interventions and diagnosis(18). Donor characteristics were manually collected from the China Organ Transplant Response Systems (CORS, www.cot.org.cn). All data were anonymized. This study is reported as per the Transparent Reporting of a Multivariable Prediction Model for Individual Prognosis or Diagnosis (TRIPOD) guidelines(19).

As a result, data of 894 cases that underwent LT from January 2015 to September 2019 were extracted. After excluding pediatric cases, simultaneous liver-kidney transplantation, living donor transplantation and cases that lack sufficient post-operative records of serum creatinine (SCr), 780cases were included. Since recipients with impaired pre-transplant renal function are prioritized during organ allocation determined by the model of end-stage liver disease (MELD) score (5), and around $90 \%$ of these patients can recover after transplantation(20), we agreed with including patients with preoperative renal injury or diagnosed with hepato-renal syndrome, out of the purpose to predict new onset AKI simply associated with perioperative treatment. As for survival analysis, the end of follow-up was set at December $31^{\text {st }}$, 2019.

Perioperative Treatment 
The grafts were procured from either donation after circulatory death (DCD), donation after brain death (DBD) or donation after brain death followed by circulatory death (DBCD)(21). No organs from executed prisoners were used. The implantation technique consisted of piggyback, standard and split liver transplantation. Liver biopsy samples were collected before and after graft reperfusion. Intraoperative extracorporeal venovenous bypass was hardly applied since it was not significantly advantageous(22). Transfusion, fluid management and use of vasoactive and hemostatic agent were adjusted according to an overall assessment of volume balance and hemodynamic stability. Boluses of vasoactive agents were mostly given to counter post-reperfusion syndrome, otherwise continuous infusion were preferred. Colloids were only used during reperfusion phase when coagulation deficiency was corrected and satisfactory urine output was observed. For patients receiving ABO-incompatible graft, Tacrolimus introduction was initiated at Day 2 after the surgery, otherwise a renal sparing therapy that initiated

Tacrolimus at Day 4 was adopted. A detailed description of anesthesia and immunotherapy can be found in Appendix 4.

\section{Outcome}

The primary outcome was postoperative AKI, diagnosed within 7 days post-operatively according to the criteria proposed by The Kidney Disease: Improving Global Outcomes (KDIGO) guideline (23) (Supplemental Content 1). Criteria concerning urine output in KDIGO guideline were not adopted, since it required urine output to be less than $0.5 \mathrm{ml} \cdot \mathrm{kg}^{-1} \cdot \mathrm{h}^{-1}$ for 6 hours to diagnose AKI, which was not as timely as the $\mathrm{SCr}$ result obtained immediately after the surgery. Moreover, for patients receiving LT we tested post-operative SCr on a daily basis, which was sufficient to identify AKI within one week after the surgery.

\section{Predictors and Selection}

A total of 111 variables were chosen for initial analysis (Appendix 1 Table 2), mainly covering demographics and donor characteristics; preoperative comorbidities, laboratory values, etiology of liver and complications; intraoperative incidents, medication, fluid infusion and blood product transfusion; post-operative medications. Certain categorical variables were generated by imposing specific rules according to their definitions (Appendix1 Table 1). MELD score was calculated according to the standard of the United Network for Organ Sharing (UNOS) Liver and Intestinal Organ Transplantation Committee (Supplemental Content 2). Graft steatosis was graded according to Nonalcoholic Steatohepatitis Clinical Research Network (NASH CRN) (https://jhuccs1.us/nash/).

For variables with a missing proportion less than $10 \%$, we imputed categorical variables with the mode and continuous variable with Multivariate Imputation By Chained Equations (MICE) algorithm(24). To minimize potential over-fitting brought by high dimensionality of the features, only features that were statistically significant $(p<0.05)$ in univariate test were chosen and subjected to a least absolute shrinkage and selection operator (LASSO) regression approach. Finally, features with non-zero coefficients after LASSO regression were used to build our models. 
Data cleaning were conducted using Python (Anaconda Distribution, Version 3.7) package. Pandas and Numpy.Scikit-learn囚https://github.com/scikit-learn/scikit-learn囚package was used to build base models including logistic regression (LR), support vector machine (SVM), random forest (RF), gradient boosting machine (GBM) implemented by decision tree and adaptive boosting (ADA). We also calculated Kalisvaart's AKI prediction score that use donor and recipient body mass index (BMI), DCD grafts, plasma requirements, and recipient warm ischemic time (WIT) as variables for risk stratification (Appendix 2).

The complete set was randomly separated into $70 \%$ training set and $30 \%$ testing set. Bootstrap method was implemented 1000 times on test set to derive confidence interval of AUC, accuracy, sensitivity and specificity. Grid search method with five-fold cross validation was used to choose best hyperparameters for each model (Appendix 2 Table 1). Mean with standard deviation, or median with interquartile range was used to analyze and express continuous variables, the comparisons of which were made using the Independent-sample $\mathrm{T}$ test or Mann-Whitney $\mathrm{U}$ test. Categorical variables were expressed in quantities and percentages and compared by the Chi-square test. Post-operative survival was estimated by KaplanMeier methods and examined by Gehan-Breslow-Wilcoxon test. SHAP method was implemented using Python shap package (https://shap.readthedocs.io/en/latest/).

\section{Results}

Baseline Characteristics of the Participants

Among the 780 cases included, 430 (55.13\%) were diagnosed with AKI (AKI group), within which 159 cases (36.97\%) were stage $3 \mathrm{AKI}$ requiring postoperative CRRT. The complete set consist of a majority of male $(n=682,87.44 \%)$, with a mean age of 50.7 years and BMl around 22.78 (Table 1$)$.

Patients that did not end up with AKI (Non-AKI group) presented comparable percentage of preoperative AKI and CKD to that of AKI group. With evident use of CRRT in AKI group (16.27\% vs. 6.85\%, p < 0.001), the biomarkers of renal function were not significantly different in clinical settings. Meanwhile, AKI group presented more severe liver dysfunction and coagulopathy, and higher MELD score (median 30 vs. 22, p< $0.001)$. AKI group also held less cases with hepatic malignancy $(28.37 \%$ vs. $54.28 \%, p<0.001)$ and higher the percent of hepatic encephalopathy (HE) $(32.33 \%$ vs. $11.7 \%, p<0.001)$. The percentage of graft steatosis and $\mathrm{ABO}$ incompatibility were also significantly higher in $\mathrm{AKI}$ group.

During LT, AKI group tended to suffer from greater blood loss and required higher volume of blood transfusion, higher dose of terlipressin, sodium bicarbonate and hemostatic medications. Consistently, the average intraoperative urine output of AKI group was significantly lower (mean $2.61 \mathrm{vs.} 3.70 \mathrm{ml} \mathrm{kg}^{-}$ $\left.{ }^{1} \cdot h^{-1}, p<0.001\right)$.

A great majority of AKI cases ( $n=288,66.97 \%$ ) were diagnosed within 24 hours after LT (Table 1$)$, that is, prior to the introduction of Tacrolimus. Although we collected data of post-operative medications prior to the appearance of diagnostic $\mathrm{SCr}$ (for AKI group) or prior to the record of maximum SCr (for Non-AKI 
group) (Appendix 3 Table 3), the heterogeneity in the timing of diagnosis made them unsuitable as predictors in our model.

The 6-month, 1-year and 2-year survival of patients in AKI group were respectively $85.52 \%, 82.65 \%$ and $79.87 \%$, which was significantly lower compared to Non-AKI group $(92.30 \%, 88.97 \%$ and $85.52 \%$ ) (Figure 1).

Feature Importance and Model Performance

Finally 14 predictors were selected (Appendix 1 Table 4) and used in each classifier to predict AKI. In 1000 bootstrap test data set, GBM model achieved the greatest $\operatorname{AUC}(0.76, \mathrm{Cl} 0.70$ to 0.82$)$, a highest F1-score $(0.73 \mathrm{Cl} 0.66$ to 0.78$)$ that tied with $\mathrm{ADA}$, and relatively balanced sensitivity $(0.74, \mathrm{Cl} 0.66$ to 0.8$)$ and specificity $(0.65, \mathrm{Cl} 0.55$ to 0.73$)$ (Figure 2). Since GBM algorithm is more robust to outliers compared to $A D A$, we eventually chose GBM model for further analysis and application.

Since Kalisvaart's AKI prediction score was built upon exclusion of patients requiring preoperative CRRT(5), we validated and compared the performance of this score and our GBM-based predictor in complete test set first, then further compared them in a subset excluding patients that received preoperative CRRT. It turned out that the AKI prediction score presented in our test set an absolutely high specificity $(1.0, \mathrm{Cl} 1.0$ to 1.0$)$ with the lowest $\mathrm{AUC}(0.52, \mathrm{Cl} 0.45$ to 0.6$)$, F1-score $(0.03, \mathrm{Cl} 0.0$ to 0.08$)$ and sensitivity $(0.02, \mathrm{Cl} 0.00$ to 0.04$)$. These metrics were not improved even in the subset excluding patients receiving preoperative CRRT. Meanwhile, GBM model also demonstrated higher AUC $(0.74, \mathrm{Cl} 0.67$ to 0.8$)$, acceptable specificity $(0.68, \mathrm{Cl} 0.59$ to 0.77$)$ and sensitivity $(0.64, \mathrm{Cl} 0.56$ to 0.73$)$ after exclusion of patients requiring pre-LT dialysis.

SHAP Values and Plots

The baseline for the Shapley value in our study is the average of all predicted AKI incidence in the test set, which was $52.08 \%$.In our test set containing 234 cases, 163 cases were correctly classified. The SHAP summary plot demonstrated that preoperative IBIL, intraoperative urine output, time under general anesthesia, preoperative PLT and graft steatosis ranked the top 5 important features (Figure $3 \mathrm{~A}$ ). Both kinds of SHAP plot revealed that higher IBIL, lower urine output, lower PLT, longer anesthesia time and graft steatosis above NASH CRN 1 were associated with higher SHAP value output in GBM model, indicating higher probability of post-LT AKI (Figure 3).

Four examples of correctly classified cases (Patient No. 104, No. 208, No. 224 and No. 229) were demonstrated as SHAP decision plot and force plot in Figure 4. The SHAP decision plots simulated the path of decision along which each feature was given in a sequence according to their availability in EMRs. The force plot mainly presented the major factors that contribute to the final model output in a certain individual. These plots increased the transparency of the prediction made by GBM algorithm. An online risk calculator to further facilitate external validation can be visited at http://wb.aidcloud.cn/zssy/aki.html $\otimes$ Figure 5 . 


\section{Discussion}

Interpretation

The cause of post-LT AKI is multifaceted. Patients with end-stage liver disease tend to have preoperative intravascular volume depletion and coagulation deficiency that predispose them to greater intraoperative blood loss and low renal perfusion(25). Besides, the technique of LT involves partial or side crossclamping of venous flow above the renal vein during anhepatic phase, which contributes to renal congestion and impairs urine output. The 14 predictors incorporated in our model are mainly indicators of preoperative liver dysfunction, intraoperative volume depletion, graft quality and difficulty of the surgery. Their correlation with AKI demonstrated by SHAP summary plot fell reasonably with the pathophysiology mentioned above, adding clinical credibility to our model. We can also tell from these correlations uncovered by $\mathrm{ML}$ algorithm that optimization of potentially modifiable variables exerting high importance in predicting AKI, such as intraoperative urine output, preoperative PLT and time under anesthesia, should be given higher priority pre- and intra-operatively. For instance, higher sentinel level of urine output might be considered in patients receiving LT. As has been shown in the SHAP dependence plot, SHAP values distribution tend to be divided around an average urine output of $2.2 \mathrm{ml} /(\mathrm{kg} \cdot \mathrm{h})$, which indicates that this might be a potential threshold for physicians to intervene. On the other hand, the criteria in KDIGO guideline requires merely an urine output below $0.5 \mathrm{ml} /(\mathrm{kg} \cdot \mathrm{h})$ for at least 6 hours to diagnose AKI. Although we did not use this criteria in our research, the correlation recognized by $\mathrm{ML}$ algorithms illuminate that a higher cut-off point of intraoperative urine output may serve to remind the physicians of renal-protective intervention in advance.

Similarly, our results also indicate that higher PLT transfusion threshold and early extubation shall be preferred in patients receiving LT. Moreover, while graft steatosis of NASH CRN 1 (steatosis involving 5\% to $33 \%$ of hepatocytes) is accepted in non-urgent LT due to worldwide shortage of organ donation, it has been identified as a risk predictor of moderate importance by $M L$ algorithms. More strict preliminary graft assessment or lower tolerance in steatosis threshold may be evaluated in the upcoming studies.

Attempts to predict AKI after LT have been made by implementing either novel ML algorithms or conventional statistical technique $(5,6,9)$. Lee, $\mathrm{H}$ et al. used a total of 72 pre- and intra-operative variables and also demonstrated that GBM-based model showed best statistical performance to predict post-LT AKI (9). Nevertheless, the disparities in techniques like use of venovenous bypass and femoral artery pressure make it hard to use our data set to externally validate this model. Yin Z. et al. identified that CIT ( $>7 \mathrm{~h})$, donor WIT (>10 min), blood loss $(>2500 \mathrm{ml}), \mathrm{SCr}(>354 \mu \mathrm{mol} / \mathrm{L})$, treatment period with dopamine ( $>6$ days) and overexposure to calcineurin inhibitor ( $\mathrm{CNI})$ may be potential risk factors of AKI in Chinese liver transplantation cohort (6). Nevertheless, in our cohort we discovered that the majority of post-LT AKI cases were diagnosed during the first 24 hours postoperatively even with delayed Tacrolimus introduction. Meanwhile, a growing proportion of DBD donors without donor WIT has altered the graft characteristics of the cohort. Therefore the power in risk stratification of these factors should be reconsidered and re-analyzed. 
Finally we decided to use Kalisvaart' s AKI prediction score as a benchmark because of our similarity in statistical performance and immunosuppression therapy(5).As a result, our GBM-based predictor demonstrated higher AUC and F1-score compared to AKI prediction score, either in our original test set or the subset conforming to their criteria that excluded patients requiring preoperative CRRT. We agreed to include patients with preoperative renal injury because these patients have a high possibility of renal recovery after transplantation(20), and are likely to be elevated in the waiting list. Early identification of deterioration in renal function in these patients would be of greater value compared to patients without preoperative renal injury. Considering the preciousness of liver graft and detrimental outcomes associated with AKI, we valued model sensitivity, that is, the ability to find out as much as possible the occurrence of AKI, over model specificity. Comparing to other ML models, boosting algorithms like GBM and ADA achieved generally highest precision and sensitivity, which is consistent with their performance of other studies $(26,27)$.

\section{Limitations}

One limitation of the current study is that it is a single center study. Liver transplantation is a highly specialized and complicated technique. Only by joint effort made by multiple centers can we build a larger data set. However, multi-center validation calls for unification in feature availability and standardized perioperative treatment. The features utilized in our model are easily accessible in most transplant centers, which enable us to promote external validation with other institutions and facilitate clinical application of this model.

Another possible limitation is that the statistical metrics of our model might not be as high as those presented in similar researches $(9,28)$. However, many of these studies built their ML models upon high dimensional features, running the risk of over-fitting. After careful feature elimination, we built our predicting model with merely 14 features, aiming for practical external validation in the future. In this way it was worthy trading statistical accuracy for model applicability. Moreover, the path of decision made by our model in each individual can be illustrated as SHAP decision plot, offering richer information in feature importance or even in potential drawbacks of the model. With such visualized explanation, physicians can interpret the model output easily and timely adjust their decisions.

\section{Implications}

Our research is a solid and generalizable work to build an applicable predictor of post-LT AKI with supervised $\mathrm{ML}$, which covers the prediction of $\mathrm{AKI}$ in patients requiring preoperative renal replacement therapy. The GBM-based model we developed consists of variables with high clinical credibility that are interoperable across institutions, and demonstrates satisfactory statistical validity and reasonable relational interpretability revealed by SHAP method.

As an emerging tool of explanatory Al, SHAP method can facilitate both local and global interpretations $(12,29)$. For local interpretation, each case has its own set of SHAP values. So it can explain how each feature contributes to the prediction of a certain case, as has been illustrated in our SHAP decision plot 
and force plot, which increases transparency and helps clinicians analyze the credibility of the prediction model. For global interpretability, the aggregate value of SHAP shows the importance of each predicting variable. Compared with traditional methods to evaluate feature importance such as the weight of RF, the SHAP value holds better consistency and can present the positive or negative relationship of each predictor.

The potential application of this model lies in its integration with the EMRs system to guide early diagnosis and interventions after LT. Since the features we selected are all easily accessible right at the end of the surgery, this GBM-based predictor of post-transplant AKI would be a convenient predicting tool that can maintain transparency of the decision-making process to clinical physicians, enabling them to adjust the final decision according to their own experience.

\section{Abbreviations}




\begin{tabular}{|c|c|}
\hline Abbreviation & Full \\
\hline HCT & hematocrit \\
\hline PLT & platelets \\
\hline WBC & white blood count \\
\hline ALT & alanine transaminase \\
\hline AST & aspartate transaminase \\
\hline TBIL & total bilirubin \\
\hline DBIL & direct bilirubin \\
\hline IBIL & indirect bilirubin \\
\hline ALB & Albumin \\
\hline $\mathrm{SCr}$ & serum creatinine \\
\hline BUN & blood urea nitrogen \\
\hline PT & prothrombin time \\
\hline APTT & activated partial thromboplastin time \\
\hline FIB & Fibrinogen \\
\hline INR & international normalized ratio \\
\hline MELD & model of end-stage liver disease \\
\hline EBL & estimated blood loss \\
\hline CRRT & continuous renal replacement therapy \\
\hline AUC & area under the receiver operating characteristic curve \\
\hline ROC & receiver operating characteristic curve \\
\hline WIT & warm ischemia time \\
\hline CIT & cold ischemia time \\
\hline LOS & length of stay \\
\hline ICU & intensive care unit \\
\hline AKI & acute kidney injury \\
\hline LT & liver transplantation \\
\hline LASSO & least absolute shrinkage and selection operator \\
\hline RF & random forest \\
\hline
\end{tabular}




\begin{tabular}{ll} 
LR & logistic regression \\
\hline SVM & support vector machine \\
\hline GBM & gradient boosting machine \\
\hline ADA & adaptive boosting \\
\hline DCD & donation after circulatory death \\
\hline DBD & donation after brain death \\
\hline DBCD & donation after brain death followed by circulatory death \\
\hline SHAP & SHapley Additive explanations \\
\hline CKD & chronic kidney disease \\
\hline EMRs & electronic medical records
\end{tabular}

\section{Declarations}

Funding: This study was supported by the National Natural Science Foundation of China (Grant No. 81974296) and by Provincial Funding for Specific Scientific and Technological Programs (No. 2019A0102005), provided by the Bureau of Technology of Meizhou city, who did not participate in any process of this work.

Conflicts of interest/Competing interests: The authors declare no competing interests.

Ethics approval: This study was approved by the Ethnic Committee of the Third Affiliated Hospital of Sun Yat-sen University (NO. [2019]02-609-01), with waiver of informed consent.

Consent to participate: Not applicable.

Consent for publication: All authors approved the publication of this manuscript.

Availability of data and material: All the analyzed results during this study are included in the appendices. The datasets analysed during the current study are available from the corresponding author on reasonable request.

Code availability: The codes used in this study are all common codes in Python packages mentioned in the part of "Methods" in the manuscript.

Author's contributions: ZH, SZ and XZ designed this research. YZ, TL collected and anonymized the original data. DY and ZL designed and guided the process of data cleaning, model building and statistics. $Y Z, C C, M G, X L, T L$ defined the rules of data extraction and interpreted patient data. DY, ZW, CS, BW, XH performed data cleaning, built the machine learning models, analyzed them with SHAP method and built 
the online GBM-based predictor. YZ and DY equally contributed in writing the manuscript. All authors read and approved the final manuscript.

\section{Acknowledgments:}

We appreciate greatly for the cooperation in data processing and model building provided by Mr. Xiang Liu and his colleagues from Guangzhou AID cloud technology co., LTD. Meanwhile, we would like to thank Prof. Yang Yang and Prof. Hui Zhao from the Department of Liver Transplant of our hospital, who have authorized access to China Organ Transplant Response Systems, for their kindly help in collect and collate the data of donors. We also give our cordial gratitude to Mr. Xun Liu, the Director of the Department of Clinical Data Center of our hospital for offering statistical guidance to our analysis, and to Mr. Shilong Gao from the Department of Information of our hospital for his help in providing access to data extraction.

\section{References}

1. Barri YM, Sanchez EQ, Jennings LW, Melton LB, Hays S, Levy MF, et al. Acute kidney injury following liver transplantation: Definition and outcome. Liver Transplantation. 2009;15(5):475-83.

2. Porter CJ, Moppett IK, Juurlink I, Nightingale J, Moran CG, Devonald MA. Acute and chronic kidney disease in elderly patients with hip fracture: prevalence, risk factors and outcome with development and validation of a risk prediction model for acute kidney injury. BMC nephrology. 2017;18(1):20.

3. Thongprayoon C, Kaewput W, Thamcharoen N, Bathini T, Watthanasuntorn K, Lertjitbanjong P, et al. Incidence and Impact of Acute Kidney Injury after Liver Transplantation: A Meta-Analysis. Journal of clinical medicine. 2019;8(3).

4. Xu Z, Luo Y, Adekkanattu P, Ancker JS, Jiang G, Kiefer RC, et al. Stratified Mortality Prediction of Patients with Acute Kidney Injury in Critical Care. Studies in health technology and informatics. 2019;264:462-6.

5. Kalisvaart M, Schlegel A, Umbro I, de Haan JE, Polak WG, JN IJ, et al. The AKI Prediction Score: a new prediction model for acute kidney injury after liver transplantation. HPB : the official journal of the International Hepato Pancreato Biliary Association. 2019;21(12):1707-17.

6. Zongyi Y, Baifeng L, Funian Z, Hao L, Xin W. Risk factors of acute kidney injury after orthotopic liver transplantation in China. Scientific reports. 2017;7:41555.

7. Ren A, Li Z, Zhang X, Deng R, Ma Y. Optimal timing of initiating CRRT in patients with acute kidney injury after liver transplantation. Annals of translational medicine. 2020;8(21):1361.

8. Gameiro J, Branco T, Lopes JA. Artificial Intelligence in Acute Kidney Injury Risk Prediction. Journal of clinical medicine. 2020;9(3).

9. Lee HC, Yoon SB, Yang SM, Kim WH, Ryu HG, Jung CW, et al. Prediction of Acute Kidney Injury after Liver Transplantation: Machine Learning Approaches vs. Logistic Regression Model. Journal of clinical medicine. 2018;7(11). 
10. Jiliang Tang SAaHL. Feature selection for classification: A review. Data Classification: Algorithms and Applications: CRC Press; 2014. p. 37-64.

11. Bihorac A, Ozrazgat-Baslanti T, Ebadi A, Motaei A, Madkour M, Pardalos PM, et al. MySurgeryRisk: Development and Validation of a Machine-learning Risk Algorithm for Major Complications and Death After Surgery. Annals of surgery. 2019;269(4):652-62.

12. Lundberg SM, Nair B, Vavilala MS, Horibe M, Eisses MJ, Adams T, et al. Explainable machine-learning predictions for the prevention of hypoxaemia during surgery. Nature biomedical engineering. 2018;2(10):749-60.

13. Parikh RB, Obermeyer Z, Navathe AS. Regulation of predictive analytics in medicine. Science. 2019;363(6429):810-2.

14. Cabitza F, Zeitoun JD. The proof of the pudding: in praise of a culture of real-world validation for medical artificial intelligence. Annals of translational medicine. 2019;7(8):161.

15. Connor CW. Artificial Intelligence and Machine Learning in Anesthesiology. Anesthesiology. 2019;131(6):1346-59.

16. Lundberg SM, Erion G, Chen H, DeGrave A, Prutkin JM, Nair B, et al. From Local Explanations to Global Understanding with Explainable Al for Trees. Nature machine intelligence. 2020;2(1):56-67.

17. Deshmukh F, Merchant SS. Explainable Machine Learning Model for Predicting GI Bleed Mortality in the Intensive Care Unit. The American journal of gastroenterology. 2020.

18. Liang H, Tsui BY, Ni H, Valentim CCS, Baxter SL, Liu G, et al. Evaluation and accurate diagnoses of pediatric diseases using artificial intelligence. Nature Medicine. 2019;25(3):433-8.

19. Collins GS, Reitsma JB, Altman DG, Moons KGM. Transparent Reporting of a multivariable prediction model for Individual Prognosis Or Diagnosis (TRIPOD): The TRIPOD Statement. Annals of internal medicine. 2015;162(1):55-63.

20. Sharma P, Goodrich NP, Zhang M, Guidinger MK, Schaubel DE, Merion RM. Short-term pretransplant renal replacement therapy and renal nonrecovery after liver transplantation alone. Clinical journal of the American Society of Nephrology : CJASN. 2013;8(7):1135-42.

21. Wang H, Jiang W, Zhou Z, Long J, Li W, Fan ST. Liver transplantation in mainland China: the overview of CLTR 2011 annual scientific report. Hepatobiliary Surg Nutr. 2013;2(4):188-97.

22. Li DH, Wald R, Blum D, McArthur E, James MT, Burns KEA, et al. Predicting mortality among critically ill patients with acute kidney injury treated with renal replacement therapy: Development and validation of new prediction models. Journal of critical care. 2020;56:113-9.

23. Palevsky PM, Liu KD, Brophy PD, Chawla LS, Parikh CR, Thakar CV, et al. KDOQI US commentary on the 2012 KDIGO clinical practice guideline for acute kidney injury. American journal of kidney diseases : the official journal of the National Kidney Foundation. 2013;61(5):649-72.

24. Zhou LZ, Yang XB, Guan Y, Xu X, Tan MT, Hou FF, et al. Development and Validation of a Risk Score for Prediction of Acute Kidney Injury in Patients With Acute Decompensated Heart Failure: A Prospective Cohort Study in China. Journal of the American Heart Association. 2016;5(11). 
25. Sanchez-Pinto LN, Khemani RG. Development of a Prediction Model of Early Acute Kidney Injury in Critically III Children Using Electronic Health Record Data. Pediatric critical care medicine : a journal of the Society of Critical Care Medicine and the World Federation of Pediatric Intensive and Critical Care Societies. 2016;17(6):508-15.

26. Kendale S, Kulkarni P, Rosenberg AD, Wang J. Supervised Machine-learning Predictive Analytics for Prediction of Postinduction Hypotension. Anesthesiology. 2018;129(4):675-88.

27. Lei VJ, Luong T, Shan E, Chen X, Neuman MD, Eneanya ND, et al. Risk Stratification for Postoperative Acute Kidney Injury in Major Noncardiac Surgery Using Preoperative and Intraoperative Data. JAMA network open. 2019;2(12):e1916921.

28. Tseng PY, Chen YT, Wang CH, Chiu KM, Peng YS, Hsu SP, et al. Prediction of the development of acute kidney injury following cardiac surgery by machine learning. Critical care. 2020;24(1):478.

29. Deshmukh F, Merchant SS. Explainable Machine Learning Model for Predicting GI Bleed Mortality in the Intensive Care Unit. The American journal of gastroenterology. 2020;115(10):1657-68.

\section{Tables}

Table 1 Characteristics, diagnosis and perioperative features of current cohort 


\begin{tabular}{|c|c|c|c|c|}
\hline & $\begin{array}{l}\text { All } \\
(N=780)\end{array}$ & $\begin{array}{l}\text { Non-AKI } \\
(n=350)\end{array}$ & $\begin{array}{l}\text { AKI } \\
(n=430)\end{array}$ & $\begin{array}{l}P \\
\text { value }\end{array}$ \\
\hline Age $(y)$ & $50.719(10.638)$ & $51.051(10.433)$ & $50.449(10.808)$ & 0.295 \\
\hline Height (cm) & 167.954(9.065) & $167.734(6.428)$ & 168.134(10.753) & 0.052 \\
\hline Weight (kg) & 64.628(11.304) & $63.404(10.889)$ & 65.628(11.548) & 0.004 \\
\hline Body Mass Index & 22.782(3.574) & 22.539(3.529) & 22.98(3.602) & 0.018 \\
\hline $\begin{array}{l}\text { Preoperative LOS } \\
\text { (d) }\end{array}$ & $11(2-26)$ & $14(4-28)$ & $8(2-23)$ & 0.001 \\
\hline \multicolumn{5}{|l|}{ Diagnosis of AKI } \\
\hline No AKI & & $350.0(100.0 \%)$ & / & \\
\hline Stage 1 AKI & & / & $177.0(41.163 \%)$ & \\
\hline Stage 2 AKI & & / & $63.0(14.651 \%)$ & \\
\hline Stage $3 \mathrm{AKI}$ & & l & $190.0(44.186 \%)$ & \\
\hline $\begin{array}{l}\text { Stage } 3 \text { AKI } \\
\text { requring CRRT }\end{array}$ & & I & $159.0(36.977 \%)$ & \\
\hline $\begin{array}{l}\text { AKI diagnosis } \\
\text { during POD1 }\end{array}$ & & & 288 (66.977\%) & \\
\hline \multicolumn{5}{|l|}{$\begin{array}{l}\text { Preoperative renal } \\
\text { function }\end{array}$} \\
\hline CKD (n) & $121.0(15.513 \%)$ & $49.0(14.0 \%)$ & $72.0(16.744 \%)$ & 0.34 \\
\hline AKI (n) & $172.0(22.051 \%)$ & $67.0(19.143 \%)$ & $105.0(24.419 \%)$ & 0.093 \\
\hline HRS (n) & $33.0(4.231 \%)$ & $8.0(2.286 \%)$ & $25.0(5.814 \%)$ & 0.024 \\
\hline $\operatorname{SCr}(\mu \mathrm{mol} / \mathrm{L})$ & $91.777(70.334)$ & $92.388(68.852)$ & 91.28(71.593) & 0.047 \\
\hline BUN (mmol/L) & $6.846(5.823)$ & $6.56(5.218)$ & 7.078(6.268) & 0.985 \\
\hline $\begin{array}{l}\text { eGFR }(\mathrm{ml} / \\
\left.\bigotimes \min * 1.73^{\wedge} 2 \bigotimes\right)\end{array}$ & $95.029(32.145)$ & $93.749(29.966)$ & 96.07(33.813) & 0.127 \\
\hline $\begin{array}{l}\text { SCr_Mean } \\
(\mu \mathrm{mol} / \mathrm{L})\end{array}$ & 79.343(71.641) & $75.837(65.256)$ & 82.197(76.402) & 0.917 \\
\hline Use of CRRT (n) & $94.0(12.051 \%)$ & $24.0(6.857 \%)$ & 70.0(16.279\%) & $<0.001$ \\
\hline $\begin{array}{l}\text { Frequency of CRRT } \\
\text { (times) }\end{array}$ & $2.567(10.727)$ & $1.351(8.312)$ & $3.556(12.269)$ & $<0.001$ \\
\hline $\begin{array}{l}\text { Preoperative } \\
\text { laboratory values }\end{array}$ & & & & \\
\hline
\end{tabular}




\begin{tabular}{|c|c|c|c|c|}
\hline HCT & $0.299(0.076)$ & $0.312(0.08)$ & $0.288(0.07)$ & $<0.001$ \\
\hline $\operatorname{PLT}\left(10^{\wedge} 9 / L\right)$ & $96.026(79.4)$ & 116.597(95.149) & 79.281(58.79) & $<0.001$ \\
\hline ALT (U/L) & $126.282(399.834)$ & $90.349(235.856)$ & 155.53(493.081) & 0.004 \\
\hline AST (U/L) & $172.242(538.996)$ & $148.429(369.227)$ & $191.626(644.817)$ & $<0.001$ \\
\hline TBIL $(\mu \mathrm{mol} / \mathrm{L})$ & $250.278(249.713)$ & $172.311(217.596)$ & $313.739(256.351)$ & $<0.001$ \\
\hline $\mathrm{DBIL}(\mu \mathrm{mol} / \mathrm{L})$ & 159.74(168.516) & $116.107(152.227)$ & 195.256(172.907) & $<0.001$ \\
\hline IBIL ( $\mu \mathrm{mol} / \mathrm{L})$ & $90.537(96.523)$ & $56.204(72.764)$ & 118.483(104.24) & $<0.001$ \\
\hline ALB (g/L) & $35.668(4.906)$ & $36.212(5.283)$ & $35.225(4.535)$ & 0.023 \\
\hline PT (s) & 25.16(13.483) & 21.115(9.851) & $28.452(15.064)$ & $<0.001$ \\
\hline APTT (s) & $54.653(20.923)$ & $49.183(16.041)$ & 59.105(23.267) & $<0.001$ \\
\hline $\mathrm{FIB}(\mathrm{g} / \mathrm{L})$ & $1.982(1.422)$ & 2.357(1.372) & $1.676(1.39)$ & $<0.001$ \\
\hline INR & $2.339(1.574)$ & $1.912(1.397)$ & $2.686(1.625)$ & $<0.001$ \\
\hline \multicolumn{5}{|l|}{ Etiology of liver } \\
\hline Hepatitis B (n) & $577.0(73.974 \%)$ & $257.0(73.429 \%)$ & $320.0(74.419 \%)$ & 0.817 \\
\hline Hepatitis C (n) & $17.0(2.179 \%)$ & $11.0(3.143 \%)$ & $6.0(1.395 \%)$ & 0.157 \\
\hline Dual infection (n) & $9.0(1.154 \%)$ & $5.0(1.429 \%)$ & $4.0(0.93 \%)$ & 0.756 \\
\hline $\begin{array}{l}\text { Hepatic } \\
\text { Malignancy (n) }\end{array}$ & $312.0(40.0 \%)$ & $190.0(54.286 \%)$ & $122.0(28.372 \%)$ & $<0.001$ \\
\hline Cirrhosis (n) & $623.0(79.872 \%)$ & $292.0(83.429 \%)$ & $331.0(76.977 \%)$ & 0.032 \\
\hline \multicolumn{5}{|l|}{$\begin{array}{l}\text { Preoperative } \\
\text { complications }\end{array}$} \\
\hline MELD score & $24(22-35)$ & $22(22-29)$ & $30(22-38)$ & $<0.001$ \\
\hline $\begin{array}{l}\text { Portal hypertension } \\
\text { (n) }\end{array}$ & $407.0(52.179 \%)$ & $192.0(54.857 \%)$ & $215.0(50.0 \%)$ & 0.201 \\
\hline Ascites (n) & $321.0(41.154 \%)$ & $142.0(40.571 \%)$ & $179.0(41.628 \%)$ & 0.822 \\
\hline HE (n) & $180.0(23.077 \%)$ & $41.0(11.714 \%)$ & $139.0(32.326 \%)$ & $<0.001$ \\
\hline Plasmapheresis (n) & $7.0(0.897 \%)$ & $2.0(0.571 \%)$ & $5.0(1.163 \%)$ & 0.625 \\
\hline HPS (n) & $4.0(0.513 \%)$ & $1.0(0.286 \%)$ & $3.0(0.698 \%)$ & 0.766 \\
\hline ARDS (n) & $7.0(0.897 \%)$ & $3.0(0.857 \%)$ & $4.0(0.93 \%)$ & 0.784 \\
\hline ALI (n) & $0.0(0.0 \%)$ & $0.0(0.0 \%)$ & $0.0(0.0 \%)$ & 1 \\
\hline $\mathrm{MV}(\mathrm{n})$ & $49.0(6.282 \%)$ & $9.0(2.571 \%)$ & $40.0(9.302 \%)$ & $<0.001$ \\
\hline
\end{tabular}




\begin{tabular}{|c|c|c|c|c|}
\hline ICU stay (n) & $439.0(56.282 \%)$ & $164.0(46.857 \%)$ & $275.0(63.953 \%)$ & $<0.001$ \\
\hline Hypernatremia (n) & $44.0(5.641 \%)$ & $10.0(2.857 \%)$ & $34.0(7.907 \%)$ & 0.004 \\
\hline $\begin{array}{l}\text { Metabolic acidosis } \\
\text { (n) }\end{array}$ & $336.0(43.077 \%)$ & $144.0(41.143 \%)$ & $192.0(44.651 \%)$ & 0.362 \\
\hline \multicolumn{5}{|l|}{$\begin{array}{l}\text { Donor } \\
\text { characteristics }\end{array}$} \\
\hline Donor age (y) & 39.191(13.966) & $38.894(14.392)$ & $39.433(13.621)$ & 0.755 \\
\hline Donor BMI & 22.578(3.199) & $22.336(3.185)$ & $22.779(3.201)$ & 0.074 \\
\hline $\begin{array}{l}\text { ABO } \\
\text { incompatibility (n) }\end{array}$ & $120.0(15.385 \%)$ & $38.0(10.857 \%)$ & $82.0(19.07 \%)$ & 0.002 \\
\hline Donor Type & & & & 0.248 \\
\hline $\mathrm{DBD}(\mathrm{n})$ & $448(57.436 \%)$ & $212(60.571 \%)$ & $236(54.884 \%)$ & \\
\hline $\mathrm{DCD}(\mathrm{n})$ & $324(41.538 \%)$ & 134(38.286\%) & $190(44.186 \%)$ & \\
\hline DBCD (n) & $8(1.026 \%)$ & $4(1.143 \%)$ & $4(0.93 \%)$ & \\
\hline $\begin{array}{l}\text { Steatosis of donor } \\
\text { liver }\end{array}$ & & & & 0.002 \\
\hline $\begin{array}{l}\text { Steatosis grade } 0 \\
\text { (n) }\end{array}$ & $529(67.821 \%)$ & $260.0(74.286 \%)$ & $269(62.558 \%)$ & \\
\hline $\begin{array}{l}\text { Steatosis grade } 1 \\
\text { (n) }\end{array}$ & $170(21.795 \%)$ & $62.0(17.714 \%)$ & $108(25.116 \%)$ & \\
\hline $\begin{array}{l}\text { Steatosis grade } 2 \\
\text { (n) }\end{array}$ & $35(4.487 \%)$ & $9.0(2.571 \%)$ & $26(6.047 \%)$ & \\
\hline $\begin{array}{l}\text { Steatosis grade } 3 \\
\text { (n) }\end{array}$ & $1(0.128 \%)$ & $0.0(0.0 \%)$ & $1(0.233 \%)$ & \\
\hline $\begin{array}{l}\text { Steatosis grade } \geq \\
1\end{array}$ & $206.0(26.41 \%)$ & $71.0(20.286 \%)$ & $135.0(31.395 \%)$ & 0.001 \\
\hline $\begin{array}{l}\text { Steatosis grade } \geq \\
2\end{array}$ & $36.0(4.615 \%)$ & $9.0(2.571 \%)$ & $27.0(6.279 \%)$ & 0.022 \\
\hline $\begin{array}{l}\text { Lack of pathology } \\
\text { assessment (n) }\end{array}$ & $45(5.769 \%)$ & $19(5.429 \%)$ & $26(6.046 \%)$ & 0.721 \\
\hline \multicolumn{5}{|l|}{$\begin{array}{l}\text { Surgery } \\
\text { characteristics }\end{array}$} \\
\hline $\begin{array}{l}\text { Time of surgery } \\
(\min )\end{array}$ & $442.713(92.854)$ & $425.297(87.949)$ & 456.888(94.418) & $<0.001$ \\
\hline $\begin{array}{l}\text { Time under } \\
\text { anesthesia (min) }\end{array}$ & $538.888(97.864)$ & $519.56(92.679)$ & $554.621(99.251)$ & $<0.001$ \\
\hline Recipient warm & $46.45(12.035)$ & $\begin{array}{l}\text { 45.919(12.183) } \\
\text { Page 18/27 }\end{array}$ & $46.883(11.909)$ & 0.088 \\
\hline
\end{tabular}


ischemic time (min)
Cold ischemic time
6.255(1.358)
6.226(1.393)
$6.278(1.329)$
0.476
(h)

Surgical technique

0.304

Piggyback (n)
Split liver (n)
Standard (n)
$\begin{aligned} & \text { Intraoperative fluid } \\ & \text { and transfusion }\end{aligned}$

\begin{tabular}{|c|c|c|c|c|}
\hline Crystalloid (ml) & $2618.423(2240.489)$ & $2775.575(2366.817)$ & 2490.944(2126.798) & 0.094 \\
\hline Colloid (ml) & $124.26(427.879)$ & $153.448(424.742)$ & $100.583(429.443)$ & 0.006 \\
\hline Albumin (ml) & 218.295(116.74) & $222.629(111.083)$ & 214.779(121.15) & 0.483 \\
\hline \multicolumn{5}{|l|}{ Transfusion } \\
\hline $\mathrm{RBC}(\mathrm{ml})$ & $1500.39(1318.45)$ & $1279.989(1333.507)$ & $1679.177(1280.024)$ & $<0.001$ \\
\hline Plasma (ml) & $1862.806(1613.71)$ & $1725.862(1376.393)$ & 1973.893(1777.029) & 0.063 \\
\hline Cryoprecipitate (U) & $30.276(15.83)$ & $27.359(14.9)$ & $32.653(16.182)$ & $<0.001$ \\
\hline $\mathrm{EBL}(\mathrm{ml})$ & $2051.489(2027.519)$ & $1679.857(1890.832)$ & 2354.685(2086.165) & $<0.001$ \\
\hline $\begin{array}{l}\text { Urine output } \\
\left(\mathrm{ml} \cdot \mathrm{kg}^{\wedge}-1 \cdot \mathrm{h}^{\wedge}-1\right)\end{array}$ & $3.104(2.146)$ & $3.708(2.219)$ & $2.613(1.954)$ & $<0.001$ \\
\hline $\begin{array}{l}\text { Ascites removal } \\
(\mathrm{ml})\end{array}$ & $959.665(1889.757)$ & 947.011(1997.938) & $969.93(1799.531)$ & 0.196 \\
\hline
\end{tabular}

\section{Intraoperative medication}

\begin{tabular}{|lllll|}
\hline rFVIla (mg) & $0.346(1.127)$ & $0.211(1.03)$ & $0.455(1.19)$ & $<0.001$ \\
\hline $\begin{array}{l}\text { Prothrombin } \\
\text { complex } \\
\text { concentrate (IU) }\end{array}$ & $587.692(433.693)$ & $554.857(434.497)$ & $614.419(431.7)$ & 0.043 \\
\hline Fibrinogen (g) & $0.404(1.293)$ & $0.342(0.735)$ & $0.453(1.609)$ & 0.567 \\
\hline Terlipressin (mg) & $0.322(0.551)$ & $0.195(0.447)$ & $0.426(0.604)$ & $<0.001$ \\
\hline $\begin{array}{l}\text { Norepinephrine, } \\
\text { bolus (mg) }\end{array}$ & $0.008(0.022)$ & $0.006(0.018)$ & $0.009(0.024)$ & 0.353 \\
\hline $\begin{array}{l}\text { Epinephrine, bolus } \\
\text { (mg) }\end{array}$ & $0.028(0.299)$ & $0.011(0.161)$ & $0.042(0.376)$ & 0.785 \\
\hline Dopamine, bolus & $12.0(1.538 \%)$ & $\begin{array}{c}4.0(1.143 \%) \\
\text { Page } 19 / 27\end{array}$ & $8.0(1.86 \%)$ & 0.874
\end{tabular}




\begin{tabular}{|lllll|}
\hline (micarbonate $(\mathrm{ml})$ & $127.006(234.266)$ & $89.429(221.225)$ & $157.593(240.316)$ & $<0.001$ \\
\hline $\begin{array}{l}\text { Use of } \\
\text { norepinephrine, } \\
\text { continuous (n) }\end{array}$ & $649.0(83.205 \%)$ & $301.0(86.0 \%)$ & $348.0(80.93 \%)$ & 0.074 \\
\hline $\begin{array}{l}\text { Use of epinephrine, } \\
\text { continuous (n) }\end{array}$ & $553.0(70.897 \%)$ & $250.0(71.429 \%)$ & $303.0(70.465 \%)$ & 0.829 \\
\hline $\begin{array}{l}\text { Use of dopamine, } \\
\text { continuous (n) }\end{array}$ & $245.0(31.41 \%)$ & $106.0(30.286 \%)$ & $139.0(32.326 \%)$ & 0.594 \\
\hline $\begin{array}{l}\text { Use of aramine (n) } \\
\text { Intraoperative }\end{array}$ & $34.0(4.359 \%)$ & $7.0(2.0 \%)$ & $27.0(6.279 \%)$ & 0.006 \\
\hline incident & & & $18.0(4.186 \%)$ & 0.008 \\
\hline Cardiac arrest (n) & $21.0(2.692 \%)$ & $3.0(0.857 \%)$ & $189.0(43.953 \%)$ & 0.108 \\
\hline Acidosis (n) & $322.0(41.282 \%)$ & $133.0(38.0 \%)$ & $351.0(81.628 \%)$ & 0.226 \\
\hline Hypotension (n) & $649.0(83.205 \%)$ & $298.0(85.143 \%)$ & & \\
\hline
\end{tabular}

$\mathrm{BMI}=$ body mass index; LOS = length of stay; $\mathrm{MELD}=$ model for end stage liver disease. CRRT = continuous renal replacement therapy; $A R D S=$ acute respiratory distress syndrome; ICU = intensive care unit; $\mathrm{HCT}$ = hematocrit; $\mathrm{PLT}$ = platelets; $\mathrm{WBC}$ = white blood cell; $\mathrm{ALT}$ = alanine transaminase; $\mathrm{AST}=$ aspartate transaminase; $\mathrm{TBIL}=$ total bilirubin; $\mathrm{DBIL}=$ direct bilirubin; $\mathrm{IBIL}=$ indirect bilirubin; $\mathrm{ALB}=$ albumin; $\mathrm{SCr}=$ serum creatinine; $\mathrm{BUN}=$ blood urea nitrogen; $\mathrm{PT}=$ prothrombin time; $\mathrm{APTT}=$ activated partial thromboplastin time; FIB = fibrinogen; INR = international normalized ratio; eGFR = estimated glomerular filtration rate; $\mathrm{DBD}=$ donation after brain death; $\mathrm{DCD}=$ donation after circulatory death; $\mathrm{DBCD}$ = donation after brain death followed by circulatory death; $\mathrm{GA}=$ general anesthesia; RBC = red blood cell; $\mathrm{EBL}=$ estimated blood loss; $r \mathrm{FVIlla}=$ recombinant activated factor VII.

\section{Figures}




\section{AKI-related Survival}

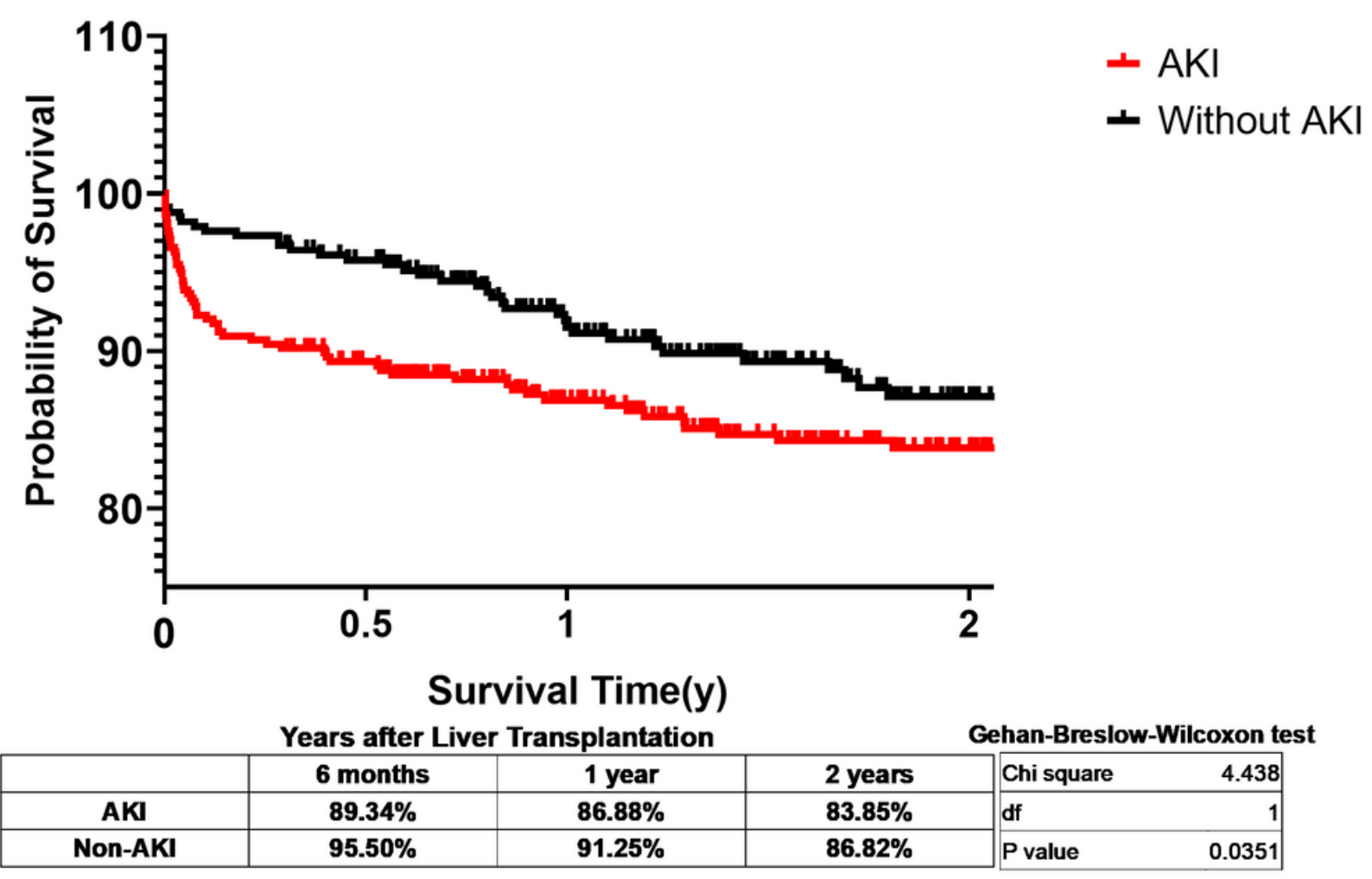

Figure 1

Postoperative survival associated with AKI. Patients with post-LT AKI demonstrated significantly lower survival, especially during the first 6 months after surgery. 


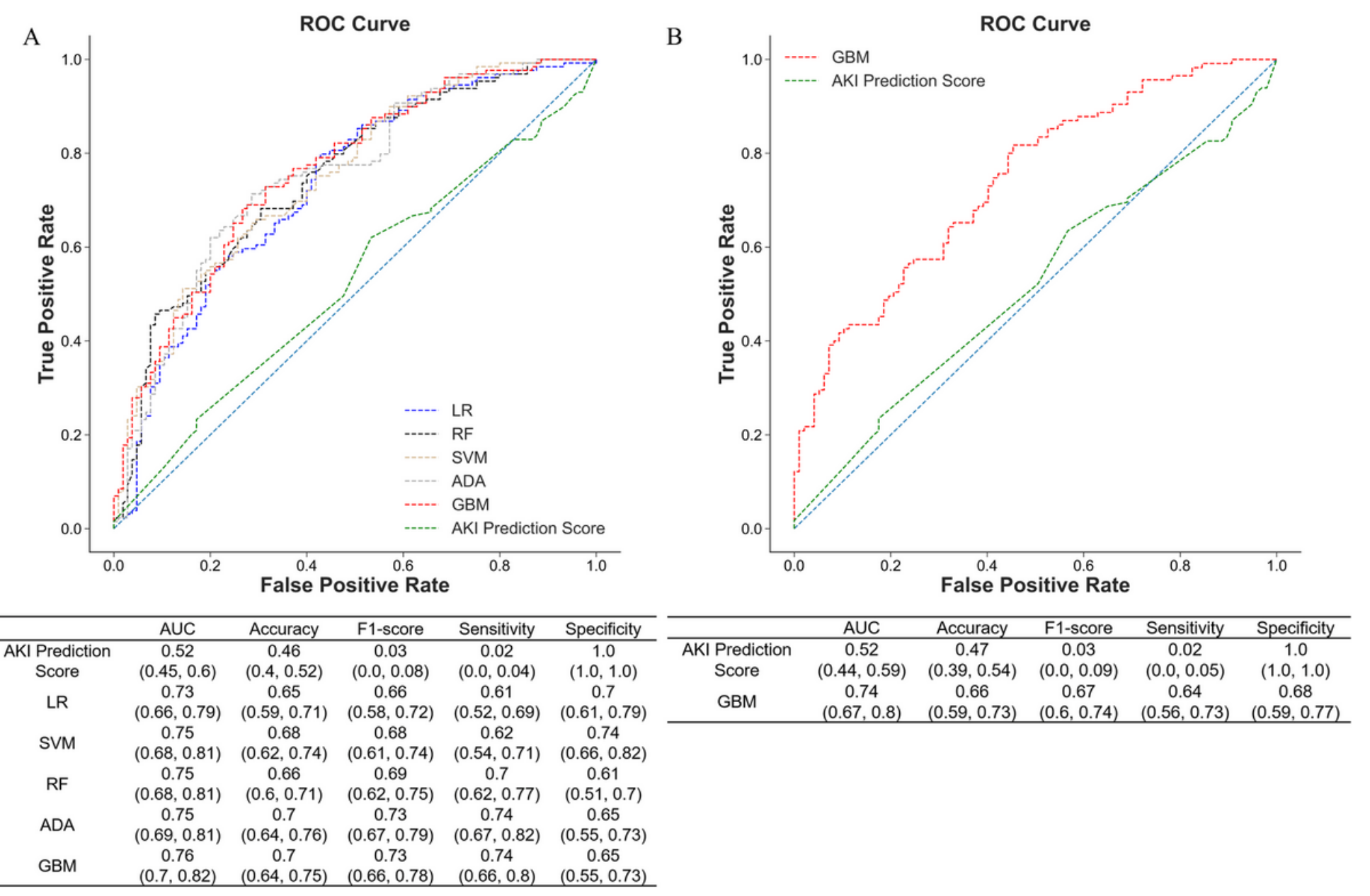

Figure 2

Performance of machine learning models and AKI prediction score. A: Performance of all predicting models in our test set, which included patients requiring preoperative CRRT. B: Performance of GBM model and AKI prediction score in a subset of our test set that excluded patients requiring preoperative CRRT, to conform to the exclusion criteria in Kalisvaart's study when they designed this score. 


\section{Shap Value Feature Importance}
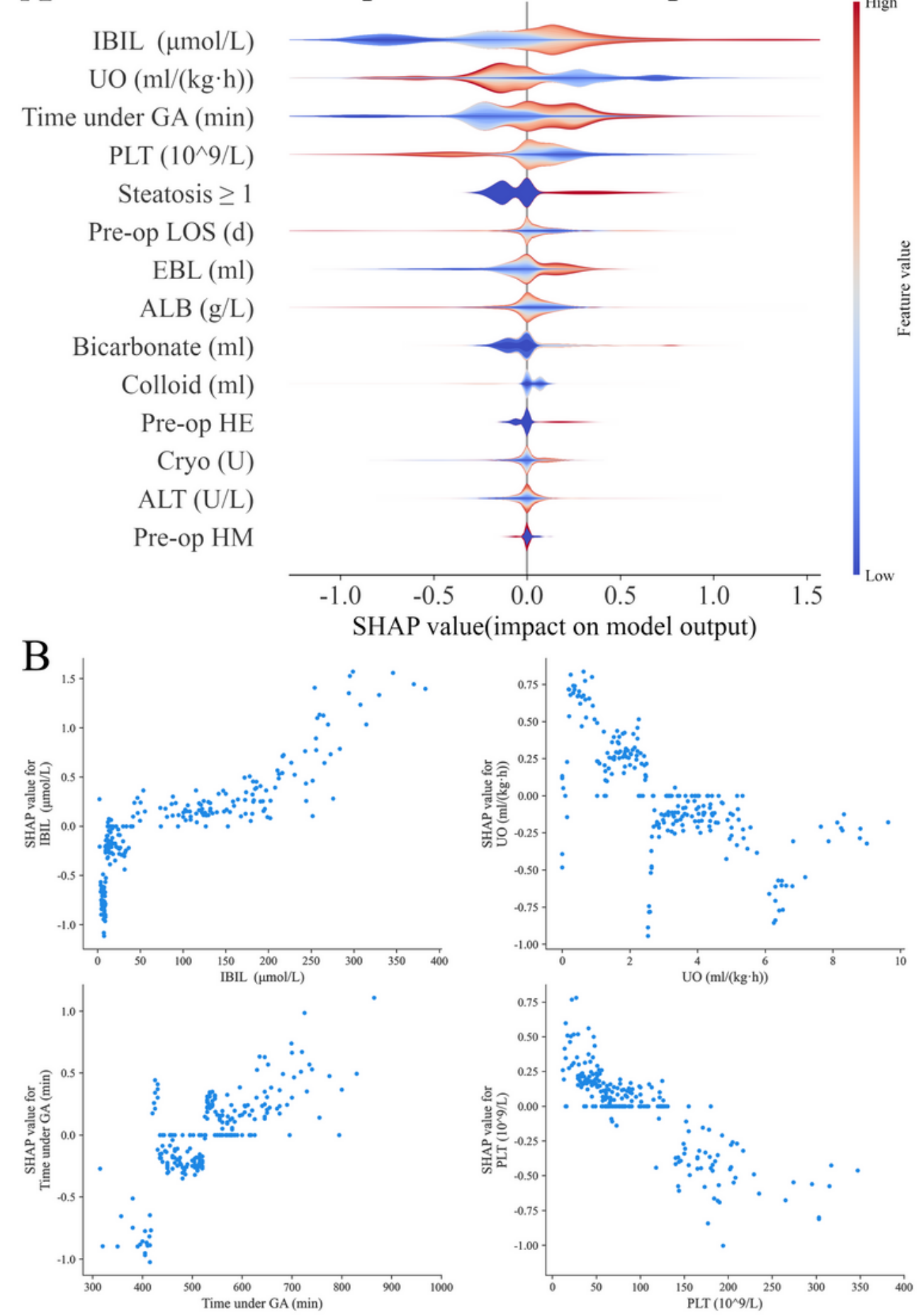

\section{Figure 3}

SHAP summary plot and dependence plot. A: The SHAP summary plot demonstrated the general importance of each feature in GBM model. The color bar on the right indicates the relative value of a feature in each case. Red dots indicate high values and blue dots indicate low values. The violin graph lining up on the midline is the aggregation of dots representing each case in the test set. The distance between the upper and lower margin of the violin graph represents the amount of the cases that end up 
with the same SHAP values offered by this feature. Categorical features including preoperative HE and HM and steatosis $\geq 1$ were represented by 0 and 1, while " 0 " means "No" and "1" means "Yes". B: SHAP dependence plot demonstrated the distribution of SHAP output value of a single feature. In our GBM prediction model, higher IBIL, lower intraoperative urine output, longer time under anesthesia and lower preoperative PLT are correlated with higher SHAP values, representing higher probability of a prediction that favors the diagnosis of AKI.

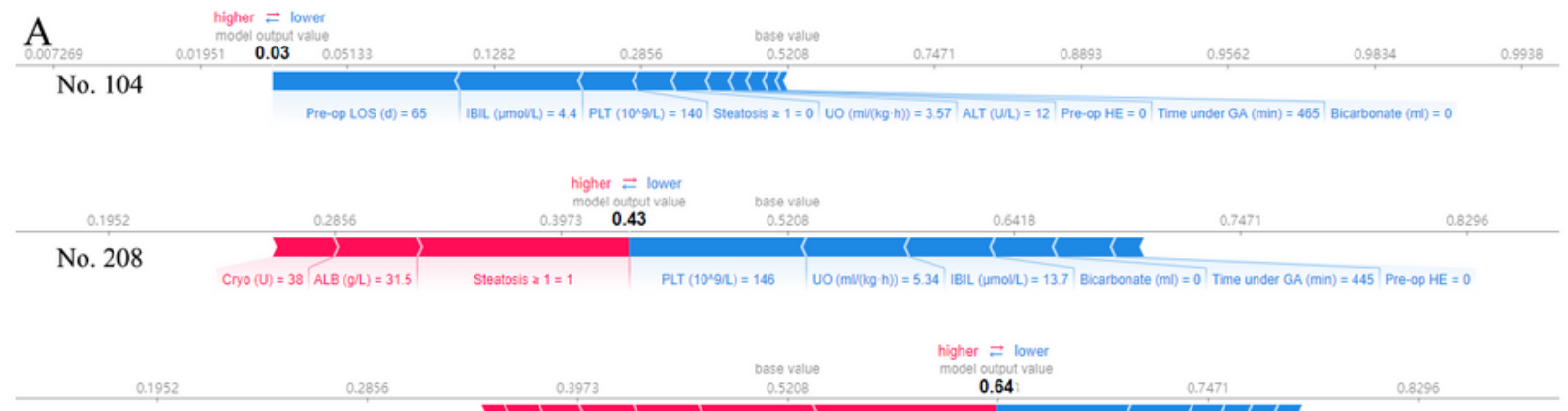

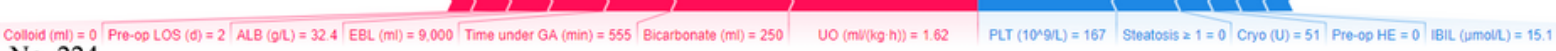
No. 224

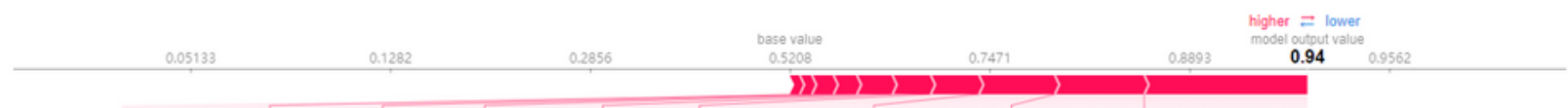
No. 229

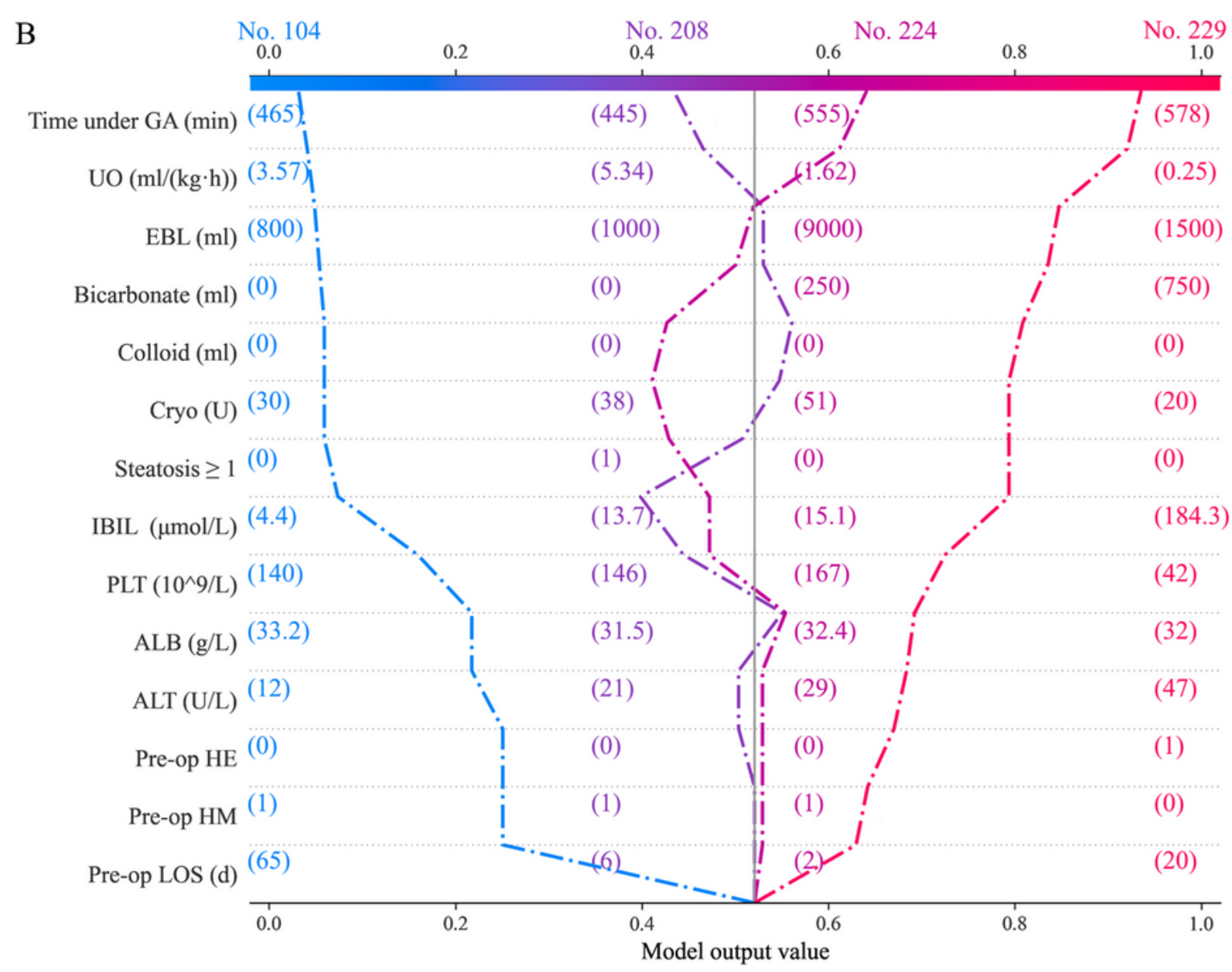

Figure 4 
SHAP decision plot and force plot A: SHAP force plots of 4 examples of patients, including patient No. 104, No 208, No. 224 and No.229. The features shown in red push the AKI probability towards the right, while the features shown in blue push the probability towards the left. This plot helps physicians to identify easily the major features with high decision power in the model on individual level. B: SHAP decision plot of the 4 patients in A. This plot is a better visualization of the feature importance of all predictors in each individual. The decision path tended to make drastic turns at feature with high importance and reached the estimated probability of AKI. Physicians can interpret the navigation made by the features and make a personal decision on the credibility of the output. 


\section{GBM-based Predictor of Post-LT AKI}

Weight $(\mathrm{kg})$

60

Platelets $\left(10^{\wedge} 9 / \mathrm{L}\right)$

140

$\operatorname{ALT}(\mathrm{U} / \mathrm{L})$

12

Hepatic malignancy $(\mathrm{Yes}=1 \mathrm{No}=0)$

1

Anesthesia on

2019-08-12 17:07:00

Colloid (ml)

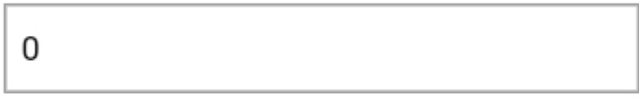

Cryoprecipitate (U)

30

Estimated blood loss (ml)

800
Preoperative length of stay (d)

65

IBIL $(\mu \mathrm{mol} / \mathrm{L})$

4.4

ALB $(\mathrm{g} / \mathrm{L})$

33.2

Hepatic encephalopathy $(\mathrm{Yes}=1 \mathrm{No}=0)$

0

Anesthesia off

2019-08-13 00:52:04

Sodium bicarbonate $(\mathrm{ml})$

0

Urine output total (ml)

1660

Steatosis grade $\geq 1(\mathrm{Yes}=1 \mathrm{No}=0)$

0

AKI(1: Positive, 0: Negative; Probability): $0(0.97)$

\section{Calculate}

\section{Figure 5}

A demo prediction of patient No.104 by online GBM-based predictor of post-LT AKI A demo prediction of patient No. 104 made by the online GBM-based predictor of post-LT AKI is shown. To increase clinical applicability, intraoperative average urine output and time of anesthesia were substituted by direct input of weight, total urine output and the time of initiation and terminal of anesthesia. The prediction output 
for patient No. 104 was " 0 " with a probability of $97 \%$, that is, the probability of this patient developing post-LT AKI was merely $3 \%$.

\section{Supplementary Files}

This is a list of supplementary files associated with this preprint. Click to download.

- Appendix1FeatureSelection.docx

- Appendix2ModeldevelopmentandSHaPsimple.docx

- Appendix3StatisticsofFeatures.docx

- Appendix4Anesthesiaandimmunotherapy.docx

- Appendix5ListofAbbreviations.docx

- SupplementalContent1KDIGOguideline.docx

- SupplementalContent2MELDiscore.docx

- graphicalabstract.tif 